\title{
Analysis of intercomponent energy transfer in the interaction of oscillating-grid turbulence with an impermeable boundary
}

Article

Accepted Version

McCorquodale, M. W. and Munro, R. J. (2018) Analysis of intercomponent energy transfer in the interaction of oscillatinggrid turbulence with an impermeable boundary. Physics of Fluids, 30 (1). 015105. ISSN 1070-6631 doi: https://doi.org/10.1063/1.5007020 Available at https://centaur.reading.ac.uk/77778/

It is advisable to refer to the publisher's version if you intend to cite from the work. See Guidance on citing.

Published version at: http://dx.doi.org/10.1063/1.5007020

To link to this article DOI: http://dx.doi.org/10.1063/1.5007020

Publisher: American Institute of Physics

All outputs in CentAUR are protected by Intellectual Property Rights law, including copyright law. Copyright and IPR is retained by the creators or other copyright holders. Terms and conditions for use of this material are defined in the End User Agreement.

www.reading.ac.uk/centaur 
Central Archive at the University of Reading

Reading's research outputs online 


\title{
Analysis of intercomponent energy transfer in the interaction of oscillating-grid turbulence with an impermeable boundary
}

\author{
Mark W. McCorquodale ${ }^{1, a)}$ and R. J. Munro ${ }^{1, b)}$ \\ Faculty of Engineering, University of Nottingham, Nottingham, NG7 2RD, UK
}

\begin{abstract}
New experimental results are presented that investigate the nature of the intercomponent energy transfer that occurs in the interaction between oscillating-grid turbulence and a solid impermeable boundary, using instantaneous velocity measurements obtained from two-dimensional particle imaging velocimetry (PIV). Estimates of the pressure-strain correlation term $\left(\Pi_{i j}^{s}\right)$ of the transport equation of the Reynolds stress tensor, which represents intercomponent energy transfer, are obtained using the PIV data from a balance of the remaining terms of the transport equation. The influence of $\Pi_{i j}^{s}$ on the flow is examined by computing the energy spectra and conditional turbulent statistics associated with events in which intercomponent energy transfer is thought to be concentrated. Data reported here is in support of viscous and 'return-to-isotropy' mechanisms governing the intercomponent energy transfer previously proposed, respectively, by Perot \& Moin [B. Perot and P. Moin, J. Fluid Mech., 295, 199-227 (1995).] and Walker et al. [D. T. Walker, R. I. Leighton and L. O. Garza-Rios, J. Fluid Mech., 320, 19-51 (1996)]. However, the data reported also indicate the presence of a weak net intercomponent energy transfer from the boundary-normal velocity component to the boundary-tangential velocity components over a thin region outside the viscous sublayer which is not captured within existing models of intercomponent energy transfer at the boundary.
\end{abstract}

\section{INTRODUCTION}

Developing an understanding of the nature of the interaction of zero-mean-shear turbulence with an impermeable boundary has proven to be a challenging problem, which has been useful in, for example, the development of turbulent models. ${ }^{1}$ This problem is of fundamental interest as it enables the direct inhibiting effects of boundaries on turbulent fluctuations to be isolated from indirect effects of the boundary (relating to the production of turbulent kinetic energy). However, despite the apparent simplified nature of this interaction, the nature of the intercomponent energy transfer that occurs within the boundary-affected region of the flow has proven to be a controversial topic. $^{2-4}$

It is thought that the initial adjustment of a zero-meanshear turbulent flow to the introduction of an impermeable boundary is well described by the rapid distortion theory (RDT) proposed by Hunt and Graham ${ }^{2}$. That is, the no-penetration condition imposed by the boundary results in a monotonic reduction in the magnitude of the boundary-normal root-mean-square (rms) turbulent velocity component, $w \equiv{\overline{\left(u_{3}^{\prime}\right)^{2}}}^{1 / 2}$, from its expected value in the absence of a boundary over a distance of approximately one integral length scale. In addition, suddenly imposing the wall-blocking condition $(w=0)$ results in pressure increases and redistribution of turbulent kinetic energy $;^{3}$ the increase in pressure instantaneously redistributes energy from the boundary-normal turbulent velocity component $(w)$ to the boundary-tangential turbulent velocity components $\left(u \equiv{\overline{\left(u_{1}^{\prime}\right)^{2}}}^{1 / 2}\right)$. This results

\footnotetext{
a)evxmm14@exmail.nottingham.ac.uk

b) rick.munro@nottingham.ac.uk
}

in amplified values of $u$ within the boundary-affected region, relative to its expected value in the absence of a boundary.

However, the subsequent development of the flow and its structure in studies in which a statistically stationary state is reached remains unclear. Results from a range of experimental and simulation based studies, conducted in both otherwise homogeneous ${ }^{3,5}$ and inhomogeneous turbulence,,${ }^{6,7}$ indicate that $w$ remains inhibited by the boundary in accordance with the RDT proposed by Hunt and Graham ${ }^{2}$. However, controversy has arisen regarding whether the blocking of $w$ continues to give rise to an intercomponent energy transfer from $w^{2}$ to $u^{2}$ through a correlation between the fluctuating pressure and velocity-strain fields, described by the so-called pressure-strain term $\Pi_{i j}^{s}$ of the Reynolds stress transport equations. That is, measurements of the pressure-strain term $\Pi_{i j}^{s}$ (in both developing and statistically stationary flow) indicate that an intercomponent energy transfer from $w^{2}$ to $u^{2}$ is significant over a thin layer, typically approximately equal in thickness to the viscous sublayer, immediately adjacent to the boundary ${ }^{3,4,7}$ but the physical interpretation of these measurements remain under dispute. That is, studies remain divided regarding whether the intercomponent energy transfer is primarily governed by dynamic viscous effects ${ }^{3}$ or by the kinematic blocking of the boundary. ${ }^{2,8,9}$

Regarding the first mechanism, Perot and Moin ${ }^{3}$ used DNS to study the effect of inserting an impermeable boundary on an otherwise isotropic and homogeneous turbulent flow and reported that, following the development of a flow to the initial insertion of the boundary, $u$ was approximately constant within the boundaryaffected region except within the viscous sublayer. Similar results for $u$ have also been reported in a range of other studies of zero-mean-shear turbulence interacting with a boundary. ${ }^{4,5,10}$ To explain these results Perot 
and Moin ${ }^{3}$ proposed a physical model in the context of "splats" and "antisplats". Here the term "splats" refers to boundary-incident turbulent motions that are blocked by the boundary; these blocking events result in high instantaneous dynamic pressure and a corresponding transfer of energy from the boundary-normal velocity component $u_{3}^{\prime}$ to the boundary-tangential velocity components $u_{1}^{\prime}$ and $u_{2}^{\prime} \cdot{ }^{3}$ The collision of fluid elements travelling tangential to the boundary was proposed to give rise to similar events, so-called "antisplats" in which the collision of elements again results in high instantaneous dynamic pressure and a transfer of energy from $u_{1}^{\prime}$ and $u_{2}^{\prime}$ to $u_{3}^{\prime}$ as the fluid elements eject from the boundary. Perot and Moin ${ }^{3}$ proposed that the energy transfer described by the pressure-strain term $\Pi_{i j}^{s}$ did not describe a net transfer of energy, but rather was a measure of the imbalance in the energy associated with "splats" and "antisplats", due to the viscous dissipation of turbulent kinetic energy (TKE) from fluid elements close to the boundary. That is, the dissipation of TKE was thought to result in antisplats that were, on average, less energetic than splats, thereby giving rise to a non-zero averaged pressure-strain term ${ }^{3}$ but which does not result in amplified values of $u$ within the boundary-affected region.

However several studies have reported that $u$ was actually amplified in the boundary-affected region relative to values expected in the absence of the boundary, ${ }^{11,12}$ which has led to an alternative interpretation of the measurements of $\Pi_{i j}^{s}$ in which it is thought that a net transfer of energy from $w^{2}$ to $u^{2}$ occurs in the boundary-affected region. Note that this interpretation is in qualitative accordance with $\mathrm{RDT}^{2}$ in which, recall, the kinematic blocking condition governs the interaction. Note, however, that in RDT the proposed amplification of $u$ is conditional on the assumption that viscous effects were confined to a viscous sublayer which was small compared to the integral length scale of the turbulence (i.e. that the Reynolds number is very high) such that viscous effects could be neglected. This assumption implies that the theory may be valid only for short times over which the turbulence does not decay significantly. As a result, the validity of RDT to describe the subsequent development of the flow after boundary insertion has been questioned. 3,5 Nonetheless, it has been proposed that RDT remains valid to describe the development of zeromean-shear turbulence at an impermeable boundary at sufficiently high Reynolds number. ${ }^{9}$

More recently Bodart, Cazalbou, and Joly ${ }^{4}$ proposed that the intercomponent energy transfer described by $\Pi_{i j}^{s}$ was indeed a net transfer of energy but which only occurs in flows with large skewness $\left(\overline{u_{3}^{\prime} u_{3}^{\prime} u_{3}^{\prime}} / w^{3}\right)$ of the turbulent velocity field. However, they were unable to provide a physical model to support their assertion that a net intercomponent energy transfer occurred. Indeed, their measured values of $u$ exhibited a monotonic reduction in the boundary-affected region - consistent with the results of Perot and Moin ${ }^{3}$, in which, we recall, it was argued that no net intercomponent energy transfer occurred.
Further from the boundary (outside the viscous sublayer but within the boundary-affected region) the sign of $\Pi_{i j}^{s}$ is reported to reverse and an intercomponent energy transfer from energy-rich $u^{2}$ components to the energy-poor $w^{2}$ component has been reported to occur. ${ }^{3,4,7,13}$ Walker, Leighton, and Garza-Rios ${ }^{13}$ proposed that this so-called "return-to-isotropy" intercomponent energy transfer results from anisotropy induced from the kinematic blocking condition acting on $u_{3}^{\prime}$. (Note that here the term "return to isotropy" is used specifically to refer to the energy transfer from $u^{2}$ to $w^{2}$, in a region in which $u>w$, and does not refer to a temporal evolution in statistics.)

In light of its statistical properties, oscillating-grid turbulence (OGT) has also been used to study the interaction of approximately zero-mean-shear turbulence at a boundary. In these studies $u$ has typically been found to be amplified in the boundary-affected region ${ }^{6,7,14,15}$ in qualitative agreement with the RDT predictions. ${ }^{2,8}$ However, in a recent study ${ }^{7}$ the current authors presented measurements of the terms of transport equation of TKE, which attributed the observed increase in $u^{2}$ primarily to the effects of turbulent transport, rather than intercomponent energy transfer. McCorquodale and Munro ${ }^{7}$ attributed the observed near-wall peak of $\Pi_{i j}^{s}$, over a thin region approximately equal in thickness to the viscous sublayer, to a viscous effect ${ }^{3}$ and consequently concluded that a net energy transfer from $w^{2}$ to $u^{2}$ (if any occurred) was not a prominent mechanism in determining the spatial structure of $u$ in the boundary-affected region of this flow. McCorquodale and Munro ${ }^{7}$ further concluded that the 'return-to-isotropy' intercomponent energy transfer was not independent of the effects of turbulent transport and that boundary-normal inhomogeneity outside the boundary-affected region could promote a stronger 'return-to-isotropy' intercomponent energy transfer within the boundary-affected region, consistent with key elements of a related model of the intercomponent energy transfer derived by Magnaudet ${ }^{9}$.

However, analysis of ongoing experiments to study the interaction of OGT with a permeable boundary (not reported here) indicates the presence of a weak net intercomponent energy transfer mechanism from $w^{2}$ to $u^{2}$ in a thin region just outside the viscous sublayer. The need to document and interpret this new observation has provided the motivation for the current paper, in which we report new results from experiments studying the interaction between OGT and a solid boundary to cast new light specifically on intercomponent energy transfer within the boundary-affected region of approximately zero-mean-shear turbulence at a solid boundary. In $\S$ II we describe the experimental set-up and the particle imaging velocimetry (PIV) technique used to measure the flow. In $\S$ III we present estimates of the pressurestrain term describing intercomponent energy transfer. In $\S I V$ we present measurements of the energy spectra which provide evidence of the nature of the intercomponent energy transfer described in $\S \mathrm{III}$. In $\S \mathrm{V}$ we present 
results of a statistical analysis of splats and antisplats which provides further evidence of the nature of the intercomponent energy transfer observed. Discussions and conclusions are made in $\S \mathrm{VI}$.

\section{EXPERIMENTS}

A schematic view of the experimental set-up is shown in figure 1. We stress that the apparatus are identical to those used in the recent related study ${ }^{7}$, to which we refer the reader for a detailed description.

The experiments were conducted in a transparent acrylic box with internal dimensions $35.2 \mathrm{~cm} \times 35.2 \mathrm{~cm} \times$ $48 \mathrm{~cm}$, henceforth denoted the 'outer box' (see figure 1), which was filled with a salt-water solution of uniform density $\rho=1.028 \mathrm{~g} / \mathrm{cm}^{3}$. (We note here that the salt water was used to make the seeding particles, need for the PIV techniques, neutrally buoyant.) An open-ended inner box, constructed from $0.5 \mathrm{~cm}$ thick transparent acrylic with internal dimensions $24.5 \mathrm{~cm} \times 24.5 \mathrm{~cm} \times 26.5 \mathrm{~cm}$, was fixed centrally on-plan at the base of the tank, as shown in figure 1, which was used to systematically reduce the mean flow present within the turbulence produced, as described in detail by McCorquodale ${ }^{16}$. We will henceforth let $2 L=24.5 \mathrm{~cm}$ denote the internal width of the inner box. The grid was suspended inside the outer box with its plane horizontal and positioned so that when at the bottom of its stroke it was $1 \mathrm{~cm}$ above the top of the inner box (see figure 1a). The grid was of stainless steel construction and consisted of an array of $7 \times 7$ bars, with square cross-section of $1 \mathrm{~cm}$ width, mesh spacing $M=5 \mathrm{~cm}$ and corresponding solidity of $36.4 \%$. The grid was attached to a stainless steel drive shaft (of $1 \mathrm{~cm}$ diameter) and vertically oscillated by converting the rotary motion of a motorised flywheel rotating with constant frequency $f$ to reciprocating linear motion using a cam and linear bearing (see figure 1a). A solid falsefloor plate, spanning the interior of the inner tank, was inserted parallel to the grid and at a depth $H \approx 4.2 M$ below the grid's mean position (see figure 1a). Previous studies have shown that at distances less than $2.5 \mathrm{M}$ below the grid's mean position the flow produced by the oscillating-grid is strongly anisotropic and exhibits coherent jet-type structures. ${ }^{16,17}$ Inserting the false-floor plate at the depth $H \approx 4.2 \mathrm{M}$ ensured that the region where the turbulence was affected by the plate did not extend into the near-grid anisotropic region.

Here, we report results from five sets of experiments, in which the stroke (defined as equal to the amplitude of the grid's motion) $S$ was set to be either $2.5 \mathrm{~cm}$ or $3.0 \mathrm{~cm}$ and the frequency of the grid's oscillation $f$ was varied between $1.6 \mathrm{~Hz}$ and $5.4 \mathrm{~Hz}$. The corresponding grid Reynolds numbers for these five experiments were $\operatorname{Re}_{G} \equiv M S f / \nu \approx 2020,4220,5260,6480$ and 8100. Further increases in $R e_{G}$ were not possible; proportionately more intense mean flows (in comparison to the magnitude of the fluctuations) are known to arise at higher grid os-

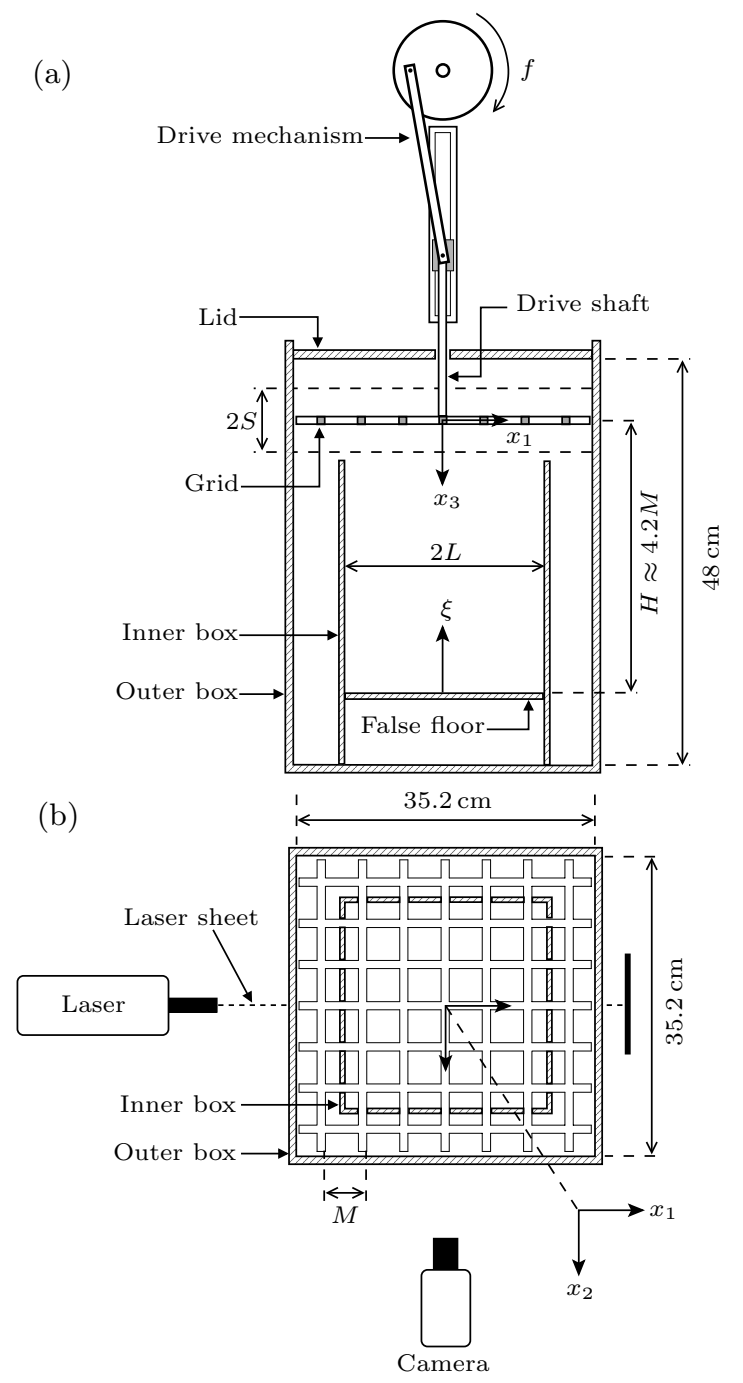

FIG. 1. Sketches showing the key components of the experimental set-up. (a) A side view showing the positioning of the reciprocating drive mechanism, the horizontal grid, the false floor and the inner and outer boxes. (b) A plan view showing the position of the inner box relative to the grid's mesh, and the position of the camera relative to the vertical laser-sheet. Also shown are the coordinate directions $\left(x_{1}, x_{2}, x_{3}\right)$, and the vertical height normal to the false floor, denoted $\xi=H-x_{3}$.

cillation frequency, ${ }^{18}$ such that turbulence produced is a poor approximation to zero-mean-shear turbulence. For each of the five grid Reynolds numbers, the experiments were repeated, under nominally identical conditions, a total of 5 times. We note that the measurements from the experiments reported at $R e_{G} \approx 2020$ and 4220 formed part of the data-set previously reported by McCorquodale and Munro ${ }^{7}$. That is, McCorquodale and Munro ${ }^{7}$ reported measurements from 31 experiments over the range $R e_{G} \approx 1520$ to 8100 , however only a subset of these experiments (at $R e_{G} \approx 2020$ and 4220 ) were repeated under nominally identical conditions. Here we have re-used this subset of the original measurements in order to report new results from the additional analysis described 
in this paper. In addition, we also report measurements from new experiments (i.e. experiments which were not reported previously in McCorquodale and Munro ${ }^{7}$ ) at $R e_{G} \approx 5260,6480$ and 8100 which were repeated a total of 5 times; this approach facilitated the use of ensembleaverages to reduce scatter in the data.

Measurements of instantaneous fluid velocities were obtained using the same technique described in McCorquodale and Munro ${ }^{7}$. The salt-water solution was seeded with neutrally buoyant tracer particles (Pliolite with diameter range 75 to $125 \mu \mathrm{m}$ ) and two-dimensional PIV applied to the vertical plane through the centre of the grid, in the region inside the inner box spanned by the grid and the false-floor. PIV calculations were performed using square interrogation windows of $13 \times 13$ pixels, overlapped to achieve 8 pixel spacing between velocity vectors, resulting in a physical spacing between velocity vectors of approximately $0.2 \mathrm{~cm}$.

The velocity data were calculated and analysed relative to the right-handed coordinate system $\left(x_{1}, x_{2}, x_{3}\right)$; here, $x_{3}$ denotes vertical depth below the mid-height of the grid's oscillation, and $\left(x_{1}, x_{2}\right)$ are the horizontal coordinates relative to the center of the grid (see figure $1)$. The corresponding velocity components are denoted $\left(u_{1}, u_{2}, u_{3}\right)$; the two components measured using the PIV set-up described above are $u_{1}\left(x_{1}, x_{3}, t\right)$ and $u_{3}\left(x_{1}, x_{3}, t\right)$, in the central plane at $x_{2}=0$. OGT is statistically stationary and so the statistical properties of the flow were analysed using time averages. That is, close to the grid (i.e. for $\left.x_{3} \lesssim 2.5 M\right)$ jets form in the wake of the grid resulting in a flow field characterised by the presence of energetic, mesh-size coherent vortex structures that interact and breakdown as they are advected away from the grid, ${ }^{16,17,19}$ such that the oscillation of the grid directly influences the structure of the flow in this region on a timescale of the order $1 / f .{ }^{16}$ However, in this study we are concerned only with measurements of the flow beneath this near-grid region (i.e. for $x_{3}>2.5 M$ ) and analysis of the velocity measurements in this region do not indicate the presence of strong signatures relating to the grid forcing frequency. Consequently, we use the conventional Reynolds decomposition $u_{i}=U_{i}+u_{i}^{\prime}$, where $u_{i}^{\prime}(\mathbf{x}, t)$ denote the fluctuating components and $U_{i}(\mathbf{x})=\overline{u_{i}}$ the time-averaged mean components (the overbar notation is used throughout to denote time averaging). Previous studies have indicated that converged time-averaged statistics derived from PIV measurements of OGT flows can be obtained using an averaging period of between $100 \mathrm{~s}$ and $120 \mathrm{~s}^{20-23}$ Here analysis of the data confirmed that the time-averaged mean and rms velocity components were converged to within approximately $5 \%$ of their ultimate values over an averaging period of $240 \mathrm{~s}$. We also introduce the coordinate $\xi=H-x_{3}$, which denotes vertical height above the false floor (see figure 1a). This coordinate is used only for convenience when plotting and comparing data; we stress that all velocities (and derivatives of velocities) were calculated in terms of the right-handed coordinates $\left(x_{1}, x_{2}, x_{3}\right)$.
In the absence of the false-floor, and away from the inner-box sidewalls, the turbulence produced by the oscillating grid was found to be in good agreement with standard models of OGT. ${ }^{17,24}$ That is, with this apparatus the turbulent flow below the anisotropic near-grid region (i.e. for $x_{3} \gtrsim 2.5 M$ ) is statistically stationary, approximately homogeneous and isotropic on horizontal planes across a central region of the tank $\left(\left|x_{1} / L\right| \leq 1 / 2\right)$ and decays spatially with distance below the grid. ${ }^{16}$ Outside the central region of the tank $\left|x_{1} / L\right| \geq 1 / 2$ anisotropic side wall regions exist, which are described in detail by McCorquodale ${ }^{16}$. Hence throughout this paper our attention is focused on the central region given by $\left|x_{1} / L\right| \leq 1 / 2$ and the sidewall anisotropic regions are ignored in the calculation of turbulent statistics. McCorquodale and Munro ${ }^{7}$ showed that, with the false-floor installed results indicate that the boundary induced effects are mostly confined to a layer of height $\delta_{s}$ above the false-floor. That is, the boundary-affected region is defined as the thin layer over which the degree of isotropy $w / u$ departs from its value of approximately unity away from the boundary ${ }^{7,17,25}$ and decreases to zero as the boundary is approached. ${ }^{4,7,18}$ It is at the edge of this region that the turbulence first feels the inhibiting effects of the kinematic blocking condition of the boundary. McCorquodale and Munro ${ }^{7}$ reported that in the current apparatus $\delta_{s}$ is of the order of the integral length scale of the turbulence, consistent with the results of previous studies. ${ }^{2,6,14}$ Data describing the statistical structure of the mean and turbulent components of the flow, both within the boundary-affected region (i.e. $\xi<\delta_{s}$ ) and above the boundary-affected region (i.e. $\xi>\delta_{s}$ ), were in good agreement with the results reported previously by McCorquodale and Munro ${ }^{7}$, to which we refer the reader for detailed illustrations of the statistical structure of the flow.

\section{ESTIMATES OF THE PRESSURE-STRAIN CORRELATION}

In section I we noted that intercomponent energy transfer is described by the pressure-strain correlation term (i.e. $\Pi_{i j}^{s}$ in equation 1 below) of the Reynolds stress transport equation, of which the steady form may be written as (see Hinze ${ }^{26}$ p. 323 )

$$
\begin{gathered}
0=\underbrace{-U_{k} \frac{\partial}{\partial x_{k}} \overline{u_{i}^{\prime} u_{j}^{\prime}}}_{A_{i j}^{d}} \underbrace{-\overline{u_{j}^{\prime} u_{k}^{\prime}} \frac{\partial U_{i}}{\partial x_{k}}-\overline{u_{i}^{\prime} u_{k}^{\prime}} \frac{\partial U_{j}}{\partial x_{k}} \underbrace{-\frac{\partial}{\partial x_{k}} \overline{u_{i}^{\prime} u_{j}^{\prime} u_{k}^{\prime}}}_{\Pi_{i j}}}_{\Phi_{i j}} \\
-\underbrace{-\frac{1}{\rho}\left(\frac{\partial}{\partial x_{i}} \overline{p^{\prime} u_{j}^{\prime}}+\frac{\partial}{\partial x_{j}} \overline{p^{\prime} u_{i}^{\prime}}\right)}_{D_{i j}}+\underbrace{\frac{1}{\rho p^{\prime}} \overline{\left(\frac{\partial u_{j}^{\prime}}{\partial x_{i}}+\frac{\partial u_{i}^{\prime}}{\partial x_{j}}\right)}}_{\Pi_{i j}^{s}} \\
+\underbrace{-2 \nu \frac{\partial u_{i}^{\prime}}{\partial x_{k}} \frac{\partial u_{j}^{\prime}}{\partial x_{k}}}_{\frac{\partial x_{k} \overline{u_{i}^{\prime} u_{j}^{\prime}}}{\partial x_{k}}},
\end{gathered}
$$


where $A_{i j}^{d}$ and $\Phi_{i j}$ denote, respectively, transport and production due to the mean flow; $T_{i j}$ and $\Pi_{i j}^{d}$ denote, respectively, transport by velocity and pressure fluctuations; $D_{i j}$ and $\varepsilon_{i j}$ denote molecular diffusion and viscous dissipation. Estimates of the terms of equation (1) for the interaction of OGT with an impermeable boundary were recently reported by the current authors. ${ }^{7}$ In this section we briefly revisit the previous estimates of $\Pi_{i j}^{s}$ reported by McCorquodale and Munro ${ }^{7}$ and highlight key aspects of the results pertinent to the current work. That is, McCorquodale and Munro ${ }^{7}$ reported estimates of $\Pi_{i j}^{s}$ from experiments which form part of the data-set reported here (i.e. those experiments at $R e_{G} \approx 2020$ and 4220). Estimates of $\Pi_{i j}^{s}$ from the experiments at $R e_{G} \approx 5260,6480$ and 8100 have not previously been reported as these experiments did not form part of the data-set initially reported in McCorquodale and Munro ${ }^{7}$ (see section II).

Within the inner box's central region - the region of interest - the turbulence is approximately homogeneous on horizontal planes and so $\overline{u_{i}^{\prime} u_{j}^{\prime}} \approx 0$ for $i \neq j$. In addition, given the redistributive nature of the pressurestrain correlation term $\left(\Pi_{i i}^{s}=0\right)$, since the turbulence is approximately homogeneous on horizontal planes we can assume $2 \Pi_{11}^{s} \approx 2 \Pi_{22}^{s} \approx-\Pi_{33}^{s}$. Hence, an understanding of the pressure-strain correlation term $\Pi_{i j}^{s}$ can be reached by considering only the transport equation for the Reynolds stress $\overline{u_{1}^{\prime} u_{1}^{\prime}}=u^{2}$. Noting that in turbulence that is approximately homogeneous on horizontal planes $\Pi_{11}^{d} \approx 0$, then the transport equation for $u^{2}$ can be expressed as

$$
\begin{gathered}
0=\underbrace{-U_{k} \frac{\partial}{\partial x_{k}} \overline{u_{1}^{\prime} u_{1}^{\prime}}}_{A_{11}^{d}} \underbrace{-2 \overline{u_{1}^{\prime} u_{k}^{\prime}} \frac{\partial U_{1}}{\partial x_{k}}}_{\Phi_{11}} \underbrace{-\frac{\partial}{\partial x_{k}} \overline{u_{1}^{\prime} u_{1}^{\prime} u_{k}^{\prime}}}_{T_{11}} \\
+\underbrace{\frac{2}{\rho p^{\prime} \frac{\partial u_{1}^{\prime}}{\partial x_{1}}}}_{\Pi_{11}^{s}}+\underbrace{\nu \frac{\partial^{2} \overline{u_{1}^{\prime} u_{1}^{\prime}}}{\partial x_{k} \partial x_{k}}}_{D_{11}} \underbrace{-2 \nu \overline{\frac{\partial u_{1}^{\prime}}{\partial x_{k}} \frac{\partial u_{1}^{\prime}}{\partial x_{k}}}}_{\varepsilon_{11}} .
\end{gathered}
$$

We stress that a direct evaluation of $\Pi_{i j}^{s}$, or the other terms of (2), is not possible for the current experiments, requiring instantaneous measurements of pressure fluctuations and of all three velocity components. However, a number of simplifying assumptions can be made to allow estimation of the terms not involving pressure fluctuations. $\Pi_{11}^{s}$ can then be estimated from a balance of the remaining terms in (2); an approach also used previously by Aronson, Johansson, and Löfdahl ${ }^{5}$. The simplifying assumptions necessary for this approach include: (i) the time-averaged statistical properties of the flow are assumed to be symmetric in the $x_{1^{-}}$and $x_{2}$-directions (based on the symmetry of the experimental set-up). (ii) The turbulence is assumed to be approximately homogeneous in horizontal planes. (iii) The approximation $\overline{\left(\partial u_{1}^{\prime} / \partial x_{2}\right)^{2}} \approx 2 \overline{\left(\partial u_{1}^{\prime} / \partial x_{1}\right)^{2}}$ can be used in calculation of the dissipation terms - a relationship which is known to hold for homogeneous, isotropic turbulence (see Pope ${ }^{27}$ p. 134). Note that when evaluating the velocity derivatives, the no slip and impermeability conditions $\left(u_{i}=0\right)$ were applied at $\xi=0$.

Estimates of the terms of (2) produced using the scheme outlined above are shown in figure 2, for experiments conducted at $R_{G} \approx 2020$ and 4220 (first reported in McCorquodale and Munro ${ }^{7}$ ), plotted against scaled height $\xi / \delta_{s}$. The scheme outlined above inevitably produced scatter in the results so, in an effort to reduce scatter, the data were averaged in the $x_{1}$-direction over the central $50 \%$ of the inner box's width (we henceforth denote this averaging by $\langle\cdot\rangle_{1}$ ) and also averaged over the 5 repeat experiments conducted under each condition. That is, as indicated in section II, previous studies using the current apparatus have identified the presence of anisotropic wall-affected regions close to the tank side walls but indicated that turbulence is approximately homogeneous on horizontal planes parallel to the grid across the central $50 \%$ of the inner box's width, ${ }^{7,16}$ consequently spatial averaging has been conducted over only this central region. To facilitate comparison, the data have been normalised by the magnitude of the corresponding component of energy dissipation evaluated at the height $\xi=\delta_{s}$, denoted $\left|\varepsilon_{11}\right|_{\delta_{s}}$. We note that here we were unable to obtain reliable estimates of the terms of (2) for experiments conducted at $R e_{G} \approx 5260,6480$ and 8100 (i.e. the additional experiments not reported in McCorquodale and Munro ${ }^{7}$ ) due to limitations inherent within our 2D PIV measurements. That is, the limited resolution of the PIV used (a resolution of approximately $0.2 \mathrm{~cm}$ ) was insufficient to capture all scales of turbulent motion and led to underestimation of some terms (primarily the dissipation $\varepsilon_{11}$ term) in equation (2). Since the pressure-strain term $\Pi_{i j}^{s}$ is determined from a balance of the remaining terms in (2) this limitation gives rise to unreliable estimates of $\Pi_{i j}^{s}$ for these experiments and, as a consequence, results for these experiments are not shown here.

Figure 2 indicates that, within the boundary-effected region, $\Pi_{11}^{s}$ initially undergoes a gradual and slight reduction over the region $0.6 \lesssim \xi / \delta_{s} \leq 1$, a slight (positive) increase over the region $0.3 \lesssim \xi / \delta_{s} \lesssim 0.6$ and a sharp increase to a near-wall (positive) peak value over the region $\xi / \delta_{s} \lesssim 0.3$. Note that the sharp increase in $\Pi_{11}^{s}$ over the region $\xi / \delta_{s} \lesssim 0.3$ occurs over a region of thickness approximately equal to the viscous sublayer. ${ }^{7}$ We stress that similar features of $\Pi_{11}^{s}$ are evident in the measurements of the studies by Perot and Moin ${ }^{3}$, Aronson, Johansson, and Löfdahl ${ }^{5}$ and Bodart, Cazalbou, and Joly ${ }^{4}$.

The initial reduction of $\Pi_{11}^{s}$ at the edge of the boundary-affected region (i.e. at $\xi / \delta_{s} \approx 1$ ) as the boundary is approached, shown in figure 2 , has previously been attributed to a return-to-isotropy intercomponent energy transfer, ${ }^{7,13}$ whilst the sharp increase in $\Pi_{11}^{s}$ over a region approximately equal in thickness to the viscous sublayer has previously been attributed to the viscous effects resulting in splat-antisplat disequilibrium. ${ }^{3,7}$ How- 
(a)
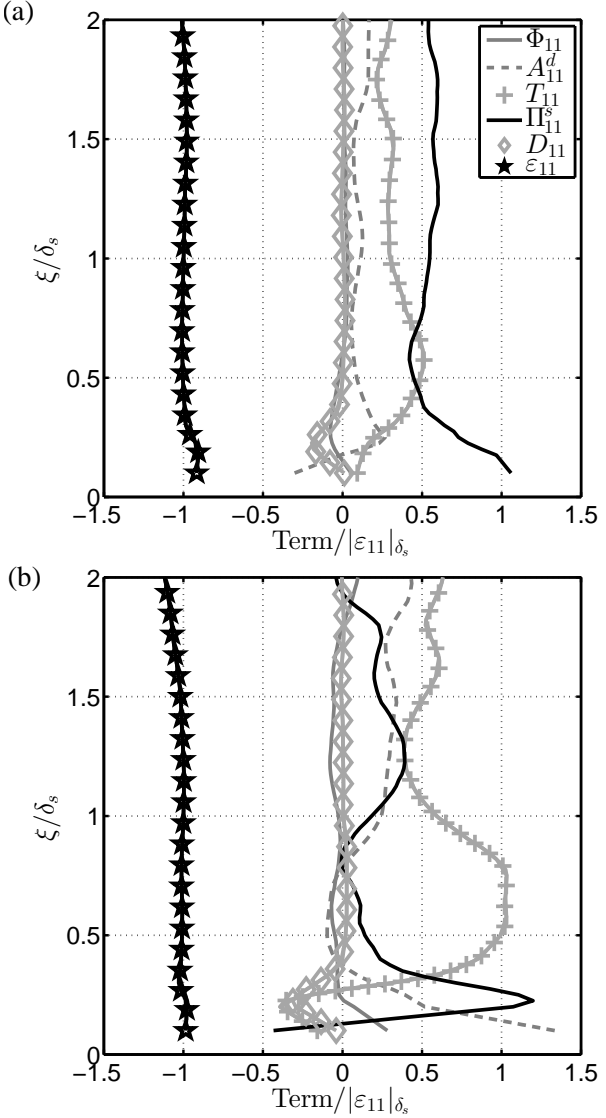

FIG. 2. Measurements of terms in the transport equation (2) at different Reynolds number - (a) $R e_{G}=2020$ and (b) $R e_{G}=4220$. Different symbols have been used to denote each term, which are shown in the legend. Each term has been spatially averaged in the $x_{1}$-direction $\left(i . e . \quad\langle\cdot\rangle_{1}\right)$, and has been normalised by the magnitude of the dissipation evaluated at the edge of the boundary-affected region, $\left|\varepsilon_{11}\right|_{\delta_{s}}$.

ever, the cause of the initial increase in $\Pi_{11}^{s}$ over the region $0.3 \lesssim \xi / \delta_{s} \lesssim 0.6$ does not appear consistent with either of these mechanisms and, as far as we are aware, has not previously received comment. Note that in previous studies of the interaction of zero-mean-shear turbulence with an impermeable boundary, ${ }^{3,4}$ the initial increases in the pressure-strain term $\Pi_{11}^{s}$ occur in regions in which viscous dissipation is not enhanced and rather the increase in $\Pi_{11}^{s}$ is offset by transport terms (as in figure 2). Consequently, this intermediate region, over which $\Pi_{11}^{s}$ is small and therefore indicates only a weak intercomponent energy transfer may occur from the boundary-normal to the boundary-tangential velocity components, is the focus of the analysis here; the interpretation of $\Pi_{11}^{s}$ over this region is explored in sections IV and V.

\section{ENERGY SPECTRA}

In previous studies of OGT interacting with a boundary, spectra have been used to gain insight into inter- component energy transfer, ${ }^{6,14,15}$ and have been compared against the spectral estimates derived from RDT. ${ }^{2}$ The energy spectra for the vertical velocity fluctuations $E_{w}\left(f_{E}\right)$ and for the horizontal velocity fluctuations $E_{u}\left(f_{E}\right)$ at different distances $\xi / \delta_{s}$ away from the plate are shown in figure 3 for a representative subset of the experiments. Here $f_{E}$ denotes the spectral frequency. Only a representative subset of the experiments has been shown in figure 3 for the purposes of brevity; we stress that the same conclusions can be drawn from the experiments at each Reynolds number. We note that, since we unable to fully resolve the dissipative scales within the flow (see section III) this may lead to a slight underestimate of the total energy content within the energy spectra. However, we stress that in this section we are primarily concerned with the effect of the boundary on the (well-resolved) large scales within the flow, such that this limitation does not alter the conclusions drawn. The estimates of the energy spectra shown in figure 3 have been normalised by the square of the magnitude of the corresponding rms turbulent velocity component at the edge of the boundary-affected region, denoted $w_{\delta_{s}}^{2}$ or $u_{\delta_{s}}^{2}$, and by the grid oscillation frequency $f$. We stress that in this flow Taylor's frozen turbulence hypothesis cannot be invoked to estimate the corresponding wavenumber spectra and that the spatial resolution of the PIV data in the $x_{1}$-direction is not sufficient to directly compute a complete wavenumber spectra. As a result, a direct analysis of the spatial scales over which the energy spectra are influenced by the boundary is not possible, nor is a quantitative comparison to the spectral estimates derived from RDT. ${ }^{2}$ Rather, in this section we are constrained to analysis within the frequency domain, from which we loosely infer results relating to the size of affected turbulent motions.

Consistent with previous work, ${ }^{2,6,14,15}$ as the boundary is approached $\left(\xi / \delta_{s} \rightarrow 0\right)$ estimates of $E_{w}$, shown in figure $3(\mathrm{a}, \mathrm{b})$, reduce at small frequencies (large scales) and depart from a $-5 / 3$ decay. [A $-5 / 3$ decay is consistent with an inertial subrange in zero-mean turbulent flow and has previously been reported in OGT. $\left.{ }^{6}\right]$ As $\xi / \delta_{s}$ reduces (see figure legend), progressively larger frequencies (smaller scales) are shown to be inhibited in qualitative agreement with the spectral estimates derived from $\mathrm{RDT},{ }^{2}$ consistent with the blocking effect of the boundary.

In contrast, estimates of $E_{u}$ shown in figure $3(\mathrm{c}, \mathrm{d})$ exhibit only very slight increases at small frequencies (large scales) as the boundary is approached (i.e as $\xi / \delta_{s}$ reduces), except within a thin region close to the boundary approximately equal in thickness to the viscous sublayer (i.e. over the region $\xi / \delta_{s} \lesssim 0.3$ ) within which $E_{u}$ diminishes as the boundary is approached as a result of the increased dissipation of TKE. (This is particularly evident in figure 3c.) The increase in $E_{u}$ at small frequencies as the boundary is approached is in qualitative accordance with previously reported measurements from OGT, ${ }^{6,14,15}$ although we note that the observed increase in $E_{u}$ is sig- 

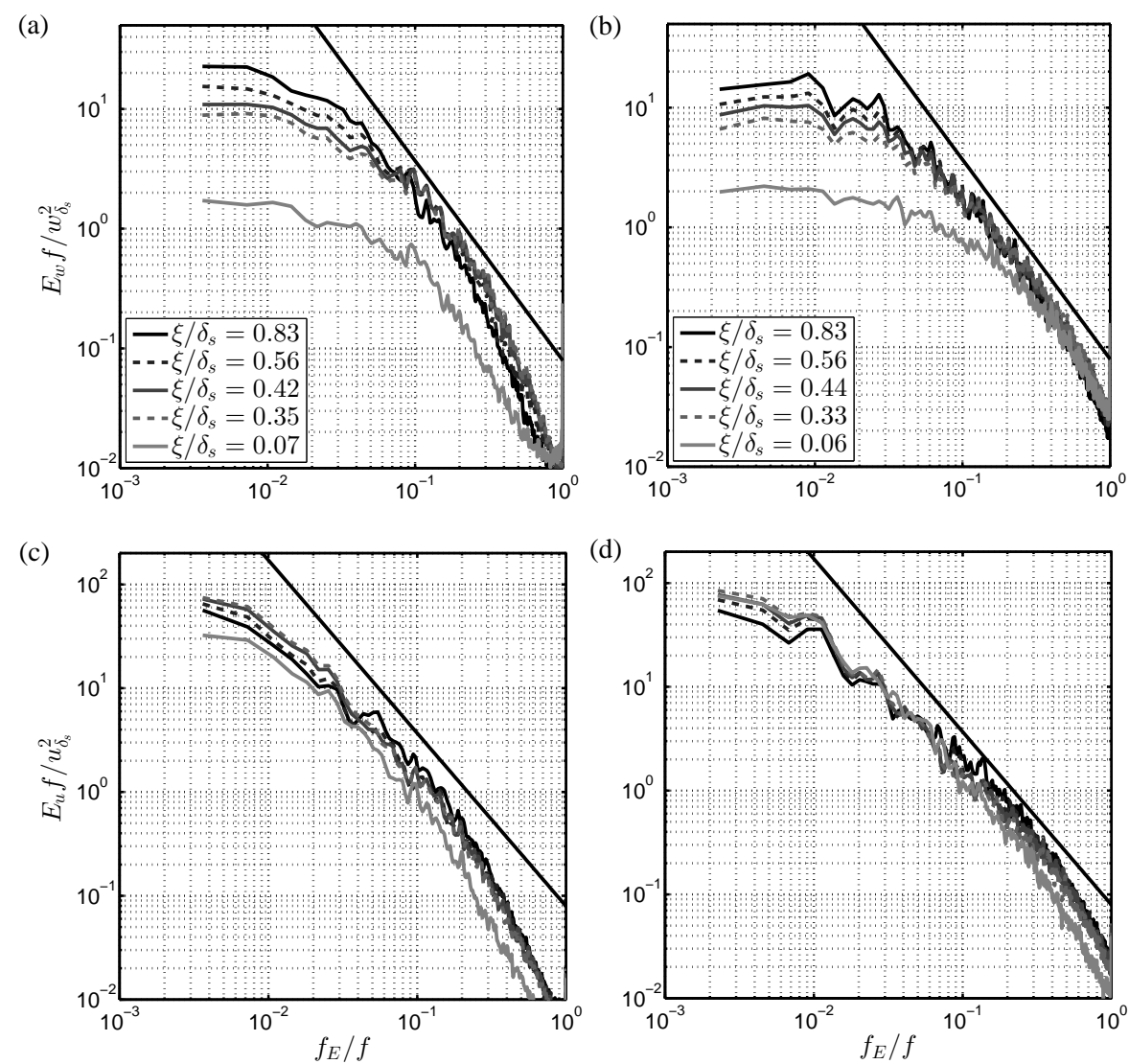

FIG. 3. Measurements of the energy spectra for (a,b) the vertical velocity fluctuations $E_{w}$ and (c,d) for the horizontal velocity fluctuations $E_{u}$ against spectral frequency $f_{E}$ for different distances above the boundary $\xi / \delta_{s}$ [see legends in (a,b) which also apply to $(\mathrm{c}, \mathrm{d})$ respectively]. Data has been normalised by the grid oscillation frequency $f$ and the magnitude of corresponding rms turbulent velocity component at the edge of the boundary-affected region, denoted $w_{\delta_{s}}^{2}$ and $u_{\delta_{s}}^{2}$. Representative results from a subset of experiments are shown; data shown in (a,c) correspond to experiments conducted at $\operatorname{Re}_{G}=4220$ and (b,d) correspond to experiments conducted at $\operatorname{Re}_{G}=8100$.

nificantly smaller than that reported by Hannoun, Fernando, and List ${ }^{6}$ for which the cause is currently unclear. This increase in $E_{u}$ at small frequencies or large scales has previously been interpreted as evidence of net intercomponent energy transfer from $w^{2}$ to $u^{2}$ at large spatial scales, sufficient to give rise to an amplification of $u^{2}$ with the boundary-affected region. ${ }^{2,6,14,15}$ In particular, amplification of the $E_{u}$ spectra at small wavenumbers has been attributed to effects of turbulent eddies impinging onto a boundary (i.e. splats), whereby they flatten at the boundary and transfer the energy of the vertical component to the horizontal components, and to the non-linear effects of vortex stretching at the boundary which act to increase $E_{u}$ at large spatial scales. ${ }^{14}$ Note however that figure 3 shows that the affect of the boundary on $E_{u}$ at small frequency is significantly smaller than the affect on $E_{w}$. This is not unexpected; some TKE is lost due to increases in the energy associated with the meandynamic pressure within the boundary-affected region. ${ }^{2}$ That is, the steady mean-flow momentum equations relate how the fluid undergoes an increase in mean dynamic pressure within the boundary-affected region at the ex- pense of TKE; for OGT under idealised conditions (zero mean flow, with the turbulence homogeneous in horizontal planes), the steady mean-flow momentum equations simplify to

$$
\frac{\partial P}{\partial x_{3}}=-\rho \frac{\partial\left(\overline{u_{3}^{\prime} u_{3}^{\prime}}\right)}{\partial x_{3}} \text { and } \frac{\partial P}{\partial x_{i}}=0, i=1,2 .
$$

Importantly, this indicates that the observed intercomponent energy transfer is not a traceless transfer of energy between the velocity components, and may only give rise to a weak amplification of $u^{2}$ within the boundaryaffected region (for example, see Perot and Moin ${ }^{3}$ and Aronson, Johansson, and Löfdahl ${ }^{5}$ ). That is, we stress that the principal mechanism reported to give rise to an amplification of $u^{2}$ within the boundary-affected region of OGT has been the blocking of a far-field TKE flux. ${ }^{7}$ Regardless, the measurements of the energy spectra are consistent with the estimates of the pressure-strain correlation term presented in section III, indicating the presence of a weak net intercomponent energy transfer from the boundary-normal to the boundary-tangential velocity component outside the viscous sublayer. 


\section{QUADRANT ANALYSIS}

In section I we noted that intercomponent energy transfer described by the pressure-strain correlation term $\Pi_{i j}^{s}$ is thought to manifest within the boundary-affected region as an imbalance in the energy associated with splats and antisplats. ${ }^{3,4}$ In this section we aim to better understand intercomponent energy transfer by use of a statistical approach to identify and isolate events within the turbulent flow that exhibit characteristics expected of splats and antisplats. That is, the stagnation flow associated with splats and antisplats is thought to exhibit strongly decelerating flow and in section $\mathrm{VA}$ we use this feature as a criteria to identify splats and antisplats. In section VB, conditional turbulent statistics associated with splats and antisplats are evaluated, where the conditions derived in section $\mathrm{VA}$ are used as sampling conditions.

\section{A. A criteria for isolating splats and antisplats}

Splats and antisplats are fluid elements that exhibit strong vertically decelerating flow (i.e. large spatial gradients in $u_{3}^{\prime}$ ); splat events moving towards the boundary $\left(u_{3}^{\prime}>0\right)$ yield negative values of the vertical strain rate $\partial u_{3}^{\prime} / \partial x_{3}$ and antisplat events moving away from the boundary $\left(u_{3}^{\prime}<0\right)$ yield positive values of the vertical strain rate $\partial u_{3}^{\prime} / \partial x_{3} \cdot{ }^{3,4,9}$ However, fluid elements that exhibit these statistical characteristics exist throughout the entire flow, as a consequence of the random structure of a turbulent flow. On the other hand, the blocking associated with the formation of splats and antisplats is expected to give rise to more strongly decelerating flow than observed in the bulk interior of the flow. Thus, it is our premise that splats and antisplats may be isolated from the background flow by use of a threshold or critical value on the magnitude of the vertical strain rate $\partial u_{3}^{\prime} / \partial x_{3}$. In the analysis that follows we define this threshold value in comparison to an average reference value of the strain rate given by $w / \bar{\ell}_{w}$, where $\bar{\ell}_{w}$ denotes the time-averaged transverse integral length scale of the boundary-normal velocity component $u_{3}^{\prime}$. This reference strain rate is devised from an order of magnitude analysis (as per Bodart, Cazalbou, and Joly ${ }^{4}$ ); when a fluid packet of size $\ell$ meets the boundary with velocity $u_{3}^{\prime}$ the vertical strain rate can be estimated as $u_{3}^{\prime} / \ell$. For a given point within the boundary-affected region the average velocity and size of a fluid packet is given by $w$ and $\bar{\ell}_{w}$ respectively; results from wavenumber spectra indicate that, within the boundary-affected region, as the boundary is approached increasingly smaller spatial scales are blocked by the boundary, ${ }^{2,14}$ such that one expects the remaining fluid packets to gradually reduce in size as the boundary is approached and, consistent with this concept, $\bar{\ell}_{w}$ monotonically reduces within the boundary-affected region as a result of the blocking effect of the boundary. ${ }^{7}$ Thus we expect a measure of the average strain rate observed within the boundary-affected region to scale with $w / \bar{\ell}_{w}$. The reference strain rate also retains an appropriate physical interpretation outside the boundary-affected region; on average we expect the vertical strain rate of the fluid packet to scale with the average packet velocity, $w$, and inversely scale with the average packet size, $\bar{\ell}_{w}$. For reference, we note that outside the boundary-affected region $\bar{\ell}_{w}$ is approximately constant and $w$ monotonically reduces as the boundary is approached. ${ }^{7}$

To validate this premise we determine typical values (ranges of high probability) of $\left|\left(\bar{\ell}_{w} / w\right) \partial u_{3}^{\prime} / \partial x_{3}\right|$ within the bulk interior of the flow and illustrate that the blocking effect of the boundary gives rise to larger values of $\left|\left(\bar{\ell}_{w} / w\right) \partial u_{3}^{\prime} / \partial x_{3}\right|$ in the boundary-affected region in comparison to those observed in the bulk interior of the flow; we interpret these measurements of strongly decelerating flow to the formation of splats and antisplats. To distinguish between splats and antisplats, we further consider measurements split into 4 quadrants based upon the sign of $u_{3}^{\prime}$ and $\partial u_{3}^{\prime} / \partial x_{3}$. That is, we calculate:

$$
\begin{gathered}
\text { Quadrant } 1\left(Q_{1}\right): \mathrm{P}_{Q_{1}} \equiv\left\langle\mathrm{P}\left(\frac{\bar{\ell}_{w}}{w} \frac{\partial u_{3}^{\prime}}{\partial x_{3}}>\phi_{T} \cap u_{3}^{\prime}>0\right)\right\rangle_{1}, \\
\text { Quadrant } 2\left(Q_{2}\right): \mathrm{P}_{Q_{2}} \equiv\left\langle\mathrm{P}\left(\frac{\bar{\ell}_{w}}{w} \frac{\partial u_{3}^{\prime}}{\partial x_{3}}>\phi_{T} \cap u_{3}^{\prime}<0\right)\right\rangle_{1}, \\
\text { Quadrant } 3\left(Q_{3}\right): \mathrm{P}_{Q_{3}} \equiv\left\langle\mathrm{P}\left(\frac{\bar{\ell}_{w}}{w} \frac{\partial u_{3}^{\prime}}{\partial x_{3}}<-\phi_{T} \cap u_{3}^{\prime}<0\right)\right\rangle_{1}, \\
\text { Quadrant } 4\left(Q_{4}\right): \mathrm{P}_{Q_{4}} \equiv\left\langle\mathrm{P}\left(\frac{\bar{\ell}_{w}}{w} \frac{\partial u_{3}^{\prime}}{\partial x_{3}}<-\phi_{T} \cap u_{3}^{\prime}>0\right)\right\rangle_{1},
\end{gathered}
$$

as a function of $\xi / \delta_{s}$, where $\mathrm{P}(A \cap B)$ denotes the probability of $A$ and $B$. Recall, $\langle\cdot\rangle_{1}$ denotes a spatial average in the $x_{1}$-direction over the central region $\left|x_{1} / L\right| \leq 1 / 2$. In equation (4) $\phi_{T}$ denotes a (positive) constant that 
is gradually increased from 0 to determine characteristic (high probability) ranges of $\left(\bar{\ell}_{w} / w\right) \partial u_{3}^{\prime} / \partial x_{3}$. That is, when $\phi_{T}=0$ we consider the probability of each quadrant based upon every measurement of the flow field, but as $\phi_{T}$ increases the evaluated probabilities associated with each quadrant decrease (i.e. $\mathrm{P}\left(\phi_{T}=0\right)>\mathrm{P}\left(\phi_{T}>\right.$ $0)$ ) because those events that exhibit $\left|\left(\bar{\ell}_{w} / w\right) \partial u_{3}^{\prime} / \partial x_{3}\right|<$ $\phi_{T}$ are excluded from the analysis. A range of values of $\phi_{T}$ have been considered but for clarity we report here only $\phi_{T}=0,2$ and 6 , which are sufficient to illustrate the key aspects of the analysis. We stress that previous work has indicated that splats and antisplats correspond to fluid elements in quadrants $Q_{4}$ and $Q_{2}$ respectively, ${ }^{3,4,9}$ therefore subsequently we consider only these quadrants.

Results from experiments at the 5 different grid Reynolds numbers considered are shown in figure 4 , plotted against scaled height $\xi / \delta_{s}$. We note that, since we unable to fully resolve the dissipative scales within the flow (see section III) this may lead to underestimates of the instantaneous vertical strain rate $\partial u_{3}^{\prime} / \partial x_{3}$. However, this is not anticipated to influence results evaluated under the condition $\phi_{T}=0$ since in this case we are concerned only with the sign of the strain rate, and not its magnitude. In addition, we stress that in this section we are primarily concerned with fluid elements as they are blocked by the boundary - a process which primarily influences the large scales within the flow (see discussion of section IV and references therein). Therefore, given that previous studies have indicated that the dissipative scales are largely unaffected by the presence of the boundary (except within the viscous sublayer) ${ }^{14}$ the small scales are not thought to be dynamically significant within this analysis; the small unresolved scales are not blocked by the boundary except within $\xi / \delta_{s} \lesssim 0.1$.

Outside of the boundary-affected region $\left(\xi / \delta_{s} \geq 1\right)$, turbulent motions do not directly feel the inhibiting effects of the boundary, so spatial gradients of $u_{3}^{\prime}$ arise only from random turbulent structure. Consequently, over this region $\left(\xi / \delta_{s} \geq 1\right)$ the overall probability (i.e. evaluated by considering every measurement of the flow field, using the condition $\phi_{T}=0$ ) of each quadrant is approximately constant with a value in the range $0.2-0.3$, shown in figure 4 . The probability that the normalised strain rate $\left(\bar{\ell}_{w} / w\right) \partial u_{3}^{\prime} / \partial x_{3}$ exceeds 2 is small (approximately 0.07 ), therefore figure 4 indicates that in this region the magnitude of the normalised strain rate typically lies between 0 and 2 . In addition, the probability that the normalised strain rate exceeds 6 is negligible. Thus we conclude that imposing the condition $\left|\left(\bar{\ell}_{w} / w\right) \partial u_{3}^{\prime} / \partial x_{3}\right| \geq 6$ appears to be sufficient to remove the background turbulent fluctuations that do not feel the inhibiting effects of the boundary.

Within the boundary-affected region $\left(\xi / \delta_{s}<1\right)$, figure 4 shows that there is a small increase in the overall probability (i.e. given by $\phi_{T}=0$ ) of events in $Q_{2}$ and $Q_{4}$ over the region $\xi / \delta_{s} \sim 0.6-1$. However, the observed magnitudes of $\partial u_{3}^{\prime} / \partial x_{3}$ over the region $0.6 \leq \xi / \delta_{s} \leq 1$ are in general consistent with those observed in the bulk
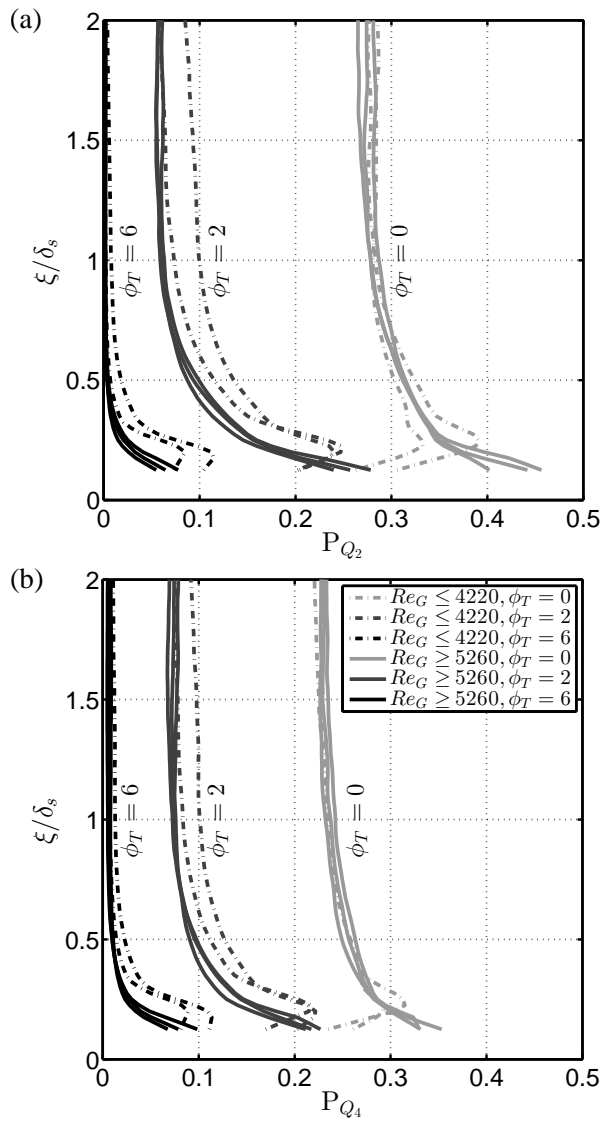

FIG. 4. Measurements of the probability (described by (4)) that the normalised strain rate $\left(\bar{\ell}_{w} / w\right) \partial u_{3}^{\prime} / \partial x_{3}$ surpasses a given constant $\phi_{T}$ [for values of $\phi_{T}$ see legend in (b) which applies to both plots], against scaled height above the boundary $\xi / \delta_{s}$. In (a) the data is subject to the additional conditions $u_{3}^{\prime}<0$ and $\partial u_{3}^{\prime} / \partial x_{3}>0$, in (b) the data is subject to the additional conditions $u_{3}^{\prime}>0$ and $\partial u_{3}^{\prime} / \partial x_{3}<0$. Results from experiments at the 5 different grid Reynolds numbers are shown for each $\phi_{T}$; in order to retain clarity within the figure each grid Reynolds number has not been individually identified, instead data are plotted differently for $R e_{G} \leq 4220$ and $R e_{G} \geq 5260$ [see legend in (b)]. Each data-set shown is an average of the measurements obtained across the 5 repeats conducted for each condition.

interior region of the flow. That is, figure 4 indicates that in the region $0.6 \leq \xi / \delta_{s} \leq 1$ the magnitude of the normalised strain rate typically still lies between 0 and 2 and the probability that an event falls within a given quadrant and that the normalised strain rate exceeds 2 is still approximately just 0.08. Crucially, the probability that the normalised strain rate exceeds 6 (i.e. the probability evaluated when $\phi_{T}=6$ ) remains negligible for $0.6 \leq \xi / \delta_{s} \leq 1$. Thus, flow that is more strongly decelerating (i.e. which exhibits a larger normalised strain rate) than the flow outside the boundary-affected region (which, recall, is indicative of splats and antisplats) is not regularly observed for $0.6 \leq \xi / \delta_{s} \leq 1$.

However, over the region $\xi / \bar{\delta}_{s} \lesssim 0.6$ the probability of events in $Q_{2}$ and $Q_{4}$ sharply increases and the observed 
magnitudes of $\partial u_{3}^{\prime} / \partial x_{3}$ of events in $Q_{2}$ and $Q_{4}$ also exceed those observed in the bulk interior region of the flow. That is, figure 4 indicates that for $\xi / \delta_{s}<0.6$ the overall probability of events in $Q_{2}$ and $Q_{4}$ (i.e. evaluated using the condition $\phi_{T}=0$ ) significantly increases as the boundary is approached. However, figure 4 also shows that for $\xi / \delta_{s}<0.6$ the probability of events in $Q_{2}$ and $Q_{4}$ when $\phi_{T}=6$ also significantly increases as the boundary is approached, and that the increase in probability when $\phi_{T}=6$ is approximately equal to the increase in probability when $\phi_{T}=0$. Thus over the region $\xi / \delta_{s}<0.6$ the increase in the probability of events in $Q_{2}$ and $Q_{4}$ is primarily driven by events which satisfy the condition $\phi_{T}=6$ (i.e. which exhibit $\left|\left(\bar{\ell}_{w} / w\right) \partial u_{3}^{\prime} / \partial x_{3}\right|>6$ ) and therefore that exhibit larger strain rates than typically observed in the bulk interior of the flow $\left(\xi / \delta_{s} \geq 1\right)$. We stress that here events in $Q_{2}$ and $Q_{4}$ exhibit spatial gradients which describe a reduction in the magnitude of $u_{3}^{\prime}$ as the boundary is approached. Hence, this increase in probability of events in $Q_{2}$ and $Q_{4}$ within the boundary-affected region (which also exhibit large normalised strain rates) is attributed to the inhibiting effects of the boundaries on the turbulence (i.e. the formation of splats and antisplats). We attribute the increasing probability of large normalised strain rates that is observed as the boundary is approached to a greater range of spatial scales being blocked by the boundary and to the prevalence of viscous effects on the turbulence close to the boundary. Thus within the boundary-affected region measured events in $Q_{2}$ and $Q_{4}$ consist of a mix of splats, antisplats and background turbulent fluctuations. However, in light of the characteristic values of $\left|\left(\bar{\ell}_{w} / w\right) \partial u_{3}^{\prime} / \partial x_{3}\right|$ observed in the bulk interior of the flow (i.e. random turbulent fluctuations are characterised by $\left.\left|\left(\bar{\ell}_{w} / w\right) \partial u_{3}^{\prime} / \partial x_{3}\right|<6\right)$, these results indicate that using the condition $\phi_{T}=6$ appears to be sufficient to isolate splats and antisplats from background turbulent fluctuations.

Note also that the results shown indicate a $R e_{G}$ effect; for $R e_{G} \leq 4220$, results, shown in figure 4, indicate that the same trends apply until $\xi / \delta_{s} \approx 0.2$, at which point the probability of events in $Q_{2}$ and $Q_{4}$ peaks and then reduces. The origin of this effect is unclear, although we conjecture that this effect may arise from a data processing issue relating to computation of the strain rates. That is, within the viscous sublayer the fluid velocities are much smaller than within the bulk of the flow, such that the uncertainty within these measurements as a proportion of the fluid velocity may be large. Thus, the error associated with these measurements may give rise to unreliable estimates of the strain rate. This effect is likely to be more prevalent at low Reynolds number as a result of the lower fluid velocities that occur in these experiments.

\section{B. Conditional statistics}

Within the boundary-affected region of the flow, intercomponent energy transfer is thought to be governed by an imbalance in the energy associated with splats and antisplats; ${ }^{3,4}$ in this section we seek to explicitly consider this imbalance by computing conditional turbulent statistics from measurements of the flow that exhibit characteristics expected of splats and antisplats. That is, we compute conditional rms values of $u_{1}^{\prime}$ where the criteria developed in section $\mathrm{VA}$ to isolate splats and antisplats are used as conditioning events. We denote the rms values of $u_{1}^{\prime}$ associated with measurements of the flow indicative of splats and antisplats by $u_{Q_{4}}$ and $u_{Q_{2}}$ respectively. Hence, $u_{Q_{2}}$ and $u_{Q_{4}}$ are rms values of $u_{1}^{\prime}$ given that the measurements used to compute these statistics satisfy the conditions $\left(\bar{\ell}_{w} / w\right) \partial u_{3}^{\prime} / \partial x_{3}>\phi_{T} \cap u_{3}^{\prime}<0$ and $\left(\bar{\ell}_{w} / w\right) \partial u_{3}^{\prime} / \partial x_{3}<-\phi_{T} \cap u_{3}^{\prime}>0$, respectively. We note that $u_{Q_{2}}\left(\phi_{T}=6\right)$ and $u_{Q_{4}}\left(\phi_{T}=6\right)$ can only be evaluated for $\xi / \delta_{s} \lesssim 0.6$ since measurements of the flow only exhibit $\left|\left(\bar{\ell}_{w} / w\right) \partial u_{3}^{\prime} / \partial x_{3}\right|>6$ (i.e. strongly decelerating flow indicative of splats and antisplats) for $\xi / \delta_{s} \lesssim 0.6$ (see section $\mathrm{VA}$ ).

In order to determine if an imbalance in the energy of splats and antisplats was dominant in giving rise to the average statistical structure of the flow within the boundary-affected region, values of $u_{Q_{2}}$ and $u_{Q_{4}}$ have been computed as a function of $\phi_{T}$. That is, conditional $u_{Q_{2}}$ and $u_{Q_{4}}$ statistics associated with specifically splats and antisplats have been computed using the critical value $\phi_{T}=6$ (see section $\mathrm{V} \mathrm{A}$ ) and the results compared against conditional statistics evaluated for $\phi_{T}<6$ (i.e. in which the effects of splats and antisplats are not isolated from the background flow). A range of values of $\phi_{T}$ have been considered but for clarity we report here only $\phi_{T}=0$ and 6 , which are sufficient to illustrate the effects of splats and antisplats, in comparison to the background flow, on the average statistical structure of the flow. That is, they are sufficient to illustrate that the imbalance of energy associated with splats and antisplats that is isolated when $\phi_{T}=6$ is able to overcome any additional weak contribution from the random turbulent fluctuations (characterised by $\left|\left(\bar{\ell}_{w} / w\right) \partial u_{3}^{\prime} / \partial x_{3}\right|<6$ ) that are included within the conditional statistics when $\phi_{T}<6$. Measurements of $u_{Q_{2}}$ and $u_{Q_{4}}$ are shown in figure 5 plotted against scaled height $\xi / \delta_{s}$.

In order to illustrate boundary effects associated with splats and antisplats on the measured values of $u_{Q_{4}}$ and $u_{Q_{2}}$, we first consider values of $u_{Q_{4}}$ and $u_{Q_{2}}$ evaluated with $\phi_{T}=0$ outside the boundary-affected region (i.e. these are values of $u_{Q_{4}}$ and $u_{Q_{2}}$ associated with the background flow only). Outside the boundary-affected region (i.e. for $\xi / \delta_{s}>1$ ) figure $5(\mathrm{a}-\mathrm{c})$ indicate that $u_{Q_{4}}>u_{Q_{2}}$, which indicates that the energy associated with turbulent motions incident towards the boundary exceeds that of turbulent motions moving away from the boundary (recall that $u_{Q_{4}}$ satisfy the condition $u_{3}^{\prime}>0$ and $u_{Q_{2}}$ the condition $u_{3}^{\prime}<0$ ). We stress that this is simply a result of the anisotropic nature of the flow produced by OGT, whereby the magnitude of turbulent fluctuations decay with distance beneath the grid, which gives rise to a TKE flux away from the grid towards the boundary. ${ }^{7}$ 

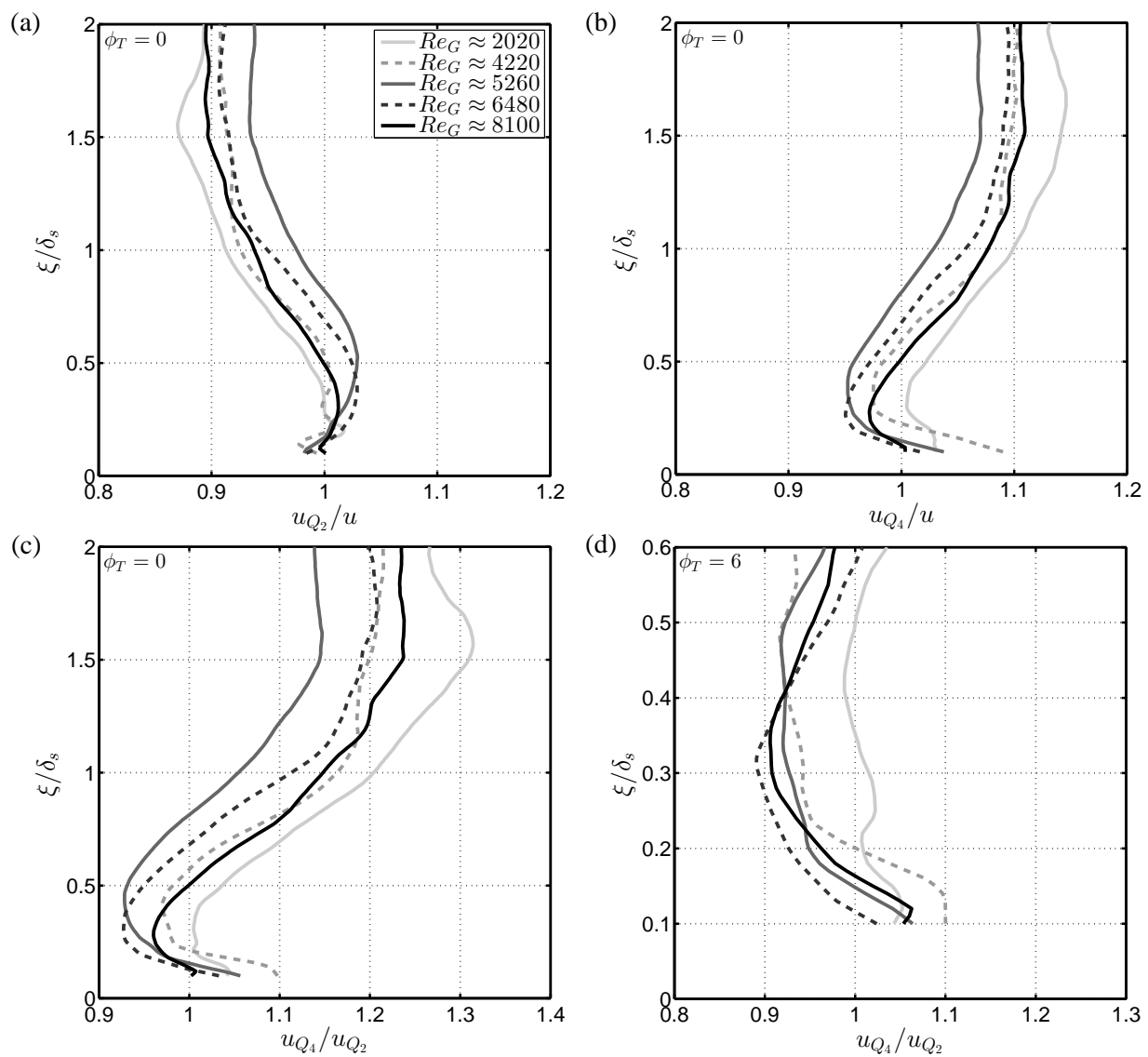

FIG. 5. Measurements (a) $u_{Q_{2}}\left(\phi_{T}=0\right)$ and $(\mathrm{b}) u_{Q_{4}}\left(\phi_{T}=0\right)$ of the conditionally sampled rms boundary-tangential velocity component plotted against scaled height above the boundary $\xi / \delta_{s}$. The data has been normalised by the rms measurements of the unsampled turbulent horizontal velocity component. (c) Shows the same data normalised to allow a direct comparison between the magnitude of $u_{Q_{2}}$ and $u_{Q_{4}}$. (d) Shows the same data as (c) subject to the condition $\phi_{T}=6$ (note the different scales used). In each plot a single data-set is shown for each experimental condition, which is an average of the measurements obtained across the 5 repeats conducted for each condition.

Figure $5(\mathrm{a}-\mathrm{c})$ show that this trend diminishes at the edge of the boundary-affected region $\left(\xi / \delta_{s} \approx 0.6\right.$ to 1.0$)$, where we can see that $u_{Q_{4}} / u_{Q_{2}}$ approaches a value of 1. Here this trend physically represents a change in the imbalance between the energy associated with turbulent motions incident towards the boundary, which are increasingly less energetic, relative to the energy of turbulent motions moving away from the boundary. Although this is evidently a boundary effect, it appears that this effect is not associated with splats and antisplats - recall that in section V A we showed that the strongly decelerating flow associated with splats and antisplats occurred only over the region $\xi / \delta_{s} \lesssim 0.6$. Instead this observation is attributed to the effects of turbulent transport, due to the blocking effect of the boundary on the boundarynormal TKE flux, ${ }^{7}$ which in isolation would act to result in $u_{Q_{4}} \approx u_{Q_{2}}$. That is, the TKE flux acts to transfer energy from energy-rich regions to energy-poor regions, such that in isolation the blocking of the TKE flux would act to give rise to constant values of $u$ within the boundary-affected region. ${ }^{7}$ In this instance there would be no imbalance, on average, between the energy associ- ated with turbulent motions incident towards the boundary and the energy of turbulent motions moving away from the boundary.

Figure $5(\mathrm{a}-\mathrm{c})$ further indicate that an imbalance in $u_{Q_{2}}$ and $u_{Q_{4}}$ is re-established as the boundary is further approached, where $u_{Q_{2}}\left(\phi_{T}=0\right)>u_{Q_{4}}\left(\phi_{T}=0\right)$ and the ratio $u_{Q_{4}} / u_{Q_{2}}$ continues to decrease over the region $0.3 \lesssim \xi / \delta_{s} \lesssim 0.6$. Hence, over this finite region the average energy associated with turbulent motions moving away from the boundary exceeds that of the energy of turbulent motions incident towards the boundary. There are two likely explanations for this trend: (i) there is a net intercomponent energy transfer from $w^{2}$ to $u^{2}$ associated with splats and antisplats over this region, or (ii) there is production and advection of TKE by the mean flow over this region. We note that the current results do depart from the zero-mean-shear condition and, therefore, effect (ii) is likely to contribute to the observed results. However, that $u_{Q_{2}}>u_{Q_{4}}$ over specifically the region $0.3 \lesssim \xi / \delta_{s} \lesssim 0.6$ is significant; recall that it was over this region that we observed significant increases in the normalised vertical strain rate $\left(\bar{\ell}_{w} / w\right) \partial u_{3}^{\prime} / \partial x_{3}$ (see 
figure 4), which was indicative of splats and antisplats in which intercomponent energy transfer is concentrated..$^{3,4}$ In addition, measurements of production and advection of TKE by the mean flow shown in figure 2 (terms $\Phi_{11}$ and $A_{11}^{d}$ ) indicate that mean flow effects are relatively small over this region.

We stress that the effect of splats and antisplats is isolated when $\phi_{T}=6$ and is shown in figure $5(\mathrm{~d})$, which also indicates that $u_{Q_{2}}>u_{Q_{4}}$ over $0.3 \lesssim \xi / \delta_{s} \lesssim 0.6$ and that the ratio $u_{Q_{4}} / u_{Q_{2}}$ also decreases over this region. Although not shown, we note that over the region $0.3 \leq \xi / \delta_{s} \leq 0.6$ the ratio $u_{Q_{4}} / u_{Q_{2}}$ gradually decreases as $\phi_{T}$ is increased from 0 to 6 , which we equate as being equivalent to gradually filtering out those motions that do not feel the inhibiting effects of the boundary. That this effect becomes more pronounced as $\phi_{T}$ increases cannot be attributed to mean flow effects (effect (ii) above), but instead is consistent with a net intercomponent energy transfer from $w^{2}$ to $u^{2}$ associated with splats and antisplats (effect (i) above) over the region $0.3 \lesssim \xi / \delta_{s} \lesssim 0.6$. Hence, we conclude not only that there is an energy transfer from $w^{2}$ to $u^{2}$ associated with splats and antisplats over this region (which can only be isolated by use of the condition $\phi_{T}=6$ ), but that this energy transfer is largely able to dominate any weak additional contribution from the random turbulent fluctuations (characterised by $\left|\left(\bar{\ell}_{w} / w\right) \partial u_{3}^{\prime} / \partial x_{3}\right|<6$ ) that are included within the conditional statistics when $\phi_{T}<6$. However, we note that a limitation of this analysis is that we are unable to derive insight into the precise dynamics that give rise to the observed splat antisplat disequilibrium and the associated intercomponent energy transfer over the region $0.3 \lesssim \xi / \delta_{s} \lesssim 0.6$

We stress that the observations over the region $0.3 \leq$ $\xi / \delta_{s} \leq 0.6$ are in contrast to the model of intercomponent energy transfer proposed by Perot and Moin ${ }^{3}$, in which the intercomponent energy transfer describes a dissipative effect whereby the average energy of splats exceeds that of antisplats. However, note that significant changes in $u_{Q_{4}}$ and $u_{Q_{2}}$ occur closer to the boundary; figure 5 indicates that $u_{Q_{4}} / u_{Q_{2}}$ increases over $\xi / \delta_{s} \lesssim 0.3$, irrespective of the value of $\phi_{T}$. That this trend occurs over $\xi / \delta_{s} \lesssim 0.3$ is significant; recall that in the current experiments this is the approximate viscous sublayer thickness within which significant dissipation of TKE occurs. ${ }^{7}$ These results physically indicate that, over this region, the energy associated with $u_{1}^{\prime} u_{1}^{\prime}$ of motions incident towards the boundary (splats) increases relative to and ultimately exceeds that of motions moving away from the boundary (antisplats). Hence, these measurements indicate that within a thin layer, approximately equal in thickness to the viscous sublayer, result are consistent with the model of Perot and Moin ${ }^{3}$, in which the imbalance between splats and antisplats is governed by the dissipation of TKE.

\section{DISCUSSION AND CONCLUSIONS}

New results from an experimental study of the interaction between oscillating-grid turbulence and a solid boundary (positioned below and aligned parallel to the grid) have been presented which investigate the nature of the intercomponent energy transfer (described by the pressure-strain correlation $\Pi_{i j}^{s}$ ) that occurs within the boundary-affected region of the flow $\left(\xi / \delta_{s} \leq 1\right)$. Within a region approximately equal in thickness to the viscous sublayer, estimates of the pressure-strain correlation $\Pi_{i j}^{s}$ indicate that an intercomponent energy transfer occurs from the boundary-normal to the boundary tangential turbulent velocity components and which promotes anisotropy. Estimates of conditional turbulent statistics also indicate that, within the region $\xi / \delta_{s} \lesssim 0.3$, intercomponent energy transfer is associated with splatantisplat disequilibrium, whereby turbulent motions incident towards the boundary are more energetic than motions away, which are characteristics of an intercomponent energy transfer primarily driven by the dissipation of TKE. ${ }^{3}$

Estimates of $\Pi_{i j}^{s}$ are shown to reduce outside the viscous sublayer, but continue to indicate a (much reduced) intercomponent energy transfer from the boundarynormal to the boundary-tangential velocity components (which also promotes anisotropy) over the region $0.3 \lesssim$ $\xi / \delta_{s} \lesssim 0.6$. In section III we noted that similar data can be found in previous studies of the interaction of zero-mean-shear turbulence with an impermeable boundary, ${ }^{3,4}$ although the results have not previously received comment. Estimates of energy spectra (section IV) were also consistent with a weak net intercomponent energy transfer from the boundary-normal to the boundary-tangential velocity components at large spatial scales; similar observations have previously been reported $^{2,6,14,15}$ which has been attributed to the effects of turbulent eddies as they impinge into a boundary (i.e splats). ${ }^{14}$ However, results indicate this is not a traceless energy transfer between the velocity components; some TKE is lost due to increases in the energy associated with the mean dynamic pressure within the boundaryaffected region. Furthermore, estimates of conditional statistics associated with splats and antisplats indicate that over this region $\left(0.3 \lesssim \xi / \delta_{s} \lesssim 0.6\right)$ the magnitude of the boundary-tangential turbulent velocity components of antisplats exceeds that of splats. Thus this splatantisplat disequilibrium is indicative of a weak net intercomponent energy transfer from the boundary-normal velocity component to the boundary-tangential velocity components, but which is not consistent with the viscous dissipative model of energy transfer proposed by Perot and Moin ${ }^{3}$ (that was prevalent in the viscous sublayer).

Instead, at first glance this energy transfer appears to be approximately in accordance with that proposed by Hunt and Graham ${ }^{2}$ for the interaction of zero-meanshear turbulence and a solid boundary. However, whilst these results share the same basic interpretation (i.e. net 
energy transfer from $w^{2}$ to $u^{2}$ ), the current results are not consistent with the model proposed by Hunt and Graham $^{2}$. That is, Hunt and Graham ${ }^{2}$ proposed that significant intercomponent energy transfer should occur only sufficiently close to the boundary - over the region $\xi / \delta_{s} \lesssim 0.3$. However, here the finite Reynolds numbers considered result in a thick viscous sublayer (of thickness $\delta_{v} / \delta_{s} \approx 0.2$ to 0.3 , where $\delta_{v}$ denotes the thickness of the viscous sublayer), such that the energy transfer proposed by Hunt and Graham ${ }^{2}$ would be inhibited by viscous effects; recall that $\mathrm{RDT}$ is valid at the limit of high Reynolds number or constant dissipation with distance above the boundary ${ }^{8,9}$ - neither of these conditions are satisfied here.

Bodart, Cazalbou, and $\mathrm{Joly}^{4}$ also proposed that a net intercomponent energy transfer from the boundarynormal to the boundary-tangential turbulent velocity components should occur and that the intensity of the energy transfer should increase as the skewness $\left(\overline{u_{3}^{\prime} u_{3}^{\prime} u_{3}^{\prime}} / w^{3}\right)$ of flow increases. Bodart, Cazalbou, and Joly ${ }^{4}$ acknowledge the role of viscosity in acting to increase the skewness of the flow close to the boundary, but argue that the imbalance in energy of splats and antisplats is set primarily by the skewness of the original velocity fields. However, a physical model consistent with the intercomponent energy transfer proposed by Bodart, Cazalbou, and Joly ${ }^{4}$ has yet to be proposed. Evidently, a large skewness associated with anisotropic flow outside the boundary-affected region (such as that reported in $\mathrm{OGT}^{7}$ ) does describe an imbalance in the energy of turbulent motions, but it is our interpretation that this cannot be used as a direct measure of splat-antisplat imbalance (by which, recall, we refer to specifically turbulent motions that form stagnation flow as a result of boundary effects) within the boundary-affected region. That is, the blocking effect of the boundary on turbulent energy fluxes associated with such anisotropic flows has a significant effect on the imbalance of energy associated with turbulent motions inside the boundary-affected region, described by the correlation coefficients $\overline{u_{3}^{\prime} u_{3}^{\prime} u_{3}^{\prime}} / w^{3}$ and $\overline{u_{3}^{\prime} u_{1}^{\prime} u_{1}^{\prime}} / w u^{2}$, which is not associated with intercomponent energy transfer. ${ }^{7}$ Indeed, despite the far-field flow here being anisotropic, the analysis of section $\mathrm{V}$ indicates splat-antisplat imbalance is actually small at the edge of the region in which we are able to identify splats and antisplats, and the imbalance only becomes significant for $\xi / \delta_{s} \leq 0.5$. Hence it appears that the current results over this region (i.e. just outside the viscous sublayer $\xi / \delta_{s} \approx 0.3$ to 0.6 ) identify a feature not captured within current key models of intercomponent energy transfer at a boundary.

A limitation in our analysis is that it does not identify an underlying physical mechanism to explain the observed intercomponent energy transfer over this region. However, so far our discussion of antisplats has focussed on regions in which the fluid ejects away from the boundary as a result of high pressure collisions of elements travelling tangential to the boundary. ${ }^{3}$ In reality, antisplats can also form without significant increases in pressure due to the interaction of two regions of opposite vorticity when a splat approaches a boundary; socalled self-generation mechanisms. ${ }^{4,28}$ That is, as a splat is blocked by the boundary, the no-slip conditions results in instantaneous shear and the vorticity associated with this shear acts to eject fluid away from the boundary. [To clarify, low pressure antisplat formation appears, in concept, similar to the process of vortex ring rebound at a solid impermeable boundary. ${ }^{29-31}$ ] We stress that these events have been reported to give rise to a notinsignificant contribution to the time-averaged measurements of the pressure-strain term. ${ }^{4}$ However, these events are not currently incorporated into key models of intercomponent energy transfer since their influence on this process is poorly understood due to their complicated dynamics. Indeed, Perot and Moin ${ }^{3}$ explicitly note that the more complicated dynamics arising in this interaction are not considered in their model in which splats and antisplats arise as simple consequences of the equation of continuity. As a result, we conjecture that these events may be a contributing factor to the weak net intercomponent energy transfer observed here.

This observed net intercomponent energy transfer from $w^{2}$ to $u^{2}$ also has implications for our understanding of the 'return-to-isotropy' model of intercomponent energy transfer; recall that Walker, Leighton, and Garza-Rios ${ }^{13}$ proposed that the anisotropy induced by the kinematic blocking condition acting on $w^{2}$ would give rise a 'returnto-isotropy' intercomponent energy transfer from $u^{2}$ to $w^{2}$. This mechanism is thought to be impeded within the viscous sublayer as a result of the prevalent viscous effects in this region. ${ }^{3,7}$ The current results further imply that this mechanism is impeded over a thin region even outside the viscous sublayer (in this study over the region $\left.0.3 \lesssim \xi / \delta_{s} \lesssim 0.6\right)$. Nevertheless, previous measurements of $\Pi_{i j}^{s}$ illustrate that this mechanism is prevalent at the edge of the boundary-affected region in the interaction of OGT with an impermeable boundary ${ }^{7}$ and in the interaction of zero-mean-shear turbulence with an impermeable boundary. ${ }^{3,4,9}$

\section{ACKNOWLEDGEMENTS}

The authors acknowledge Jim Meakin and Steve Lawton for technical support with the experimental apparatus. MWM acknowledges financial support through an Engineering and Physical Sciences Research Council Studentship (number 1510466).

${ }^{1}$ B. Perot and P. Moin, "Shear free turbulent boundary layers, Part 2, new concepts for Reynolds stress transport equation modelling of inhomogeneous flows," J. Fluid Mech. 295, 229-245 (1995).

${ }^{2}$ J. C. R. Hunt and J. M. R. Graham, "Free-stream turbulence near plane boundaries," J. Fluid Mech. 84(2), 209-235 (1978).

${ }^{3}$ B. Perot and P. Moin, "Shear free turbulent boundary layers, Part 1, physical insights into near-wall turbulence," J. Fluid Mech. 295, 199-227 (1995). 
${ }^{4}$ J. Bodart, J. B. Cazalbou, and L. Joly, "Direct numerical simulation of unsheared turbulence diffusing towards a free-slip or no-slip surface," J. Turbul. 11(48), 1-18 (2010).

${ }^{5}$ D. Aronson, A. V. Johansson, and L. Löfdahl, "Shear-free turbulence near a wall," J. Fluid Mech. 338, 363-395 (1997).

${ }^{6}$ I. A. Hannoun, H. J. S. Fernando, and E. J. List, "Turbulence structure near a sharp density interface," J. Fluid Mech. 189, 189-209 (1988).

${ }^{7}$ M. W. McCorquodale and R. J. Munro, "Experimental study of oscillating-grid turbulence interacting with a solid boundary," J. Fluid Mech. 813, 768-798 (2017).

${ }^{8}$ J. C. R. Hunt, "Turbulence structure in thermal convection and shear-free boundary layers," J. Fluid Mech. 138, 161-184 (1984).

${ }^{9}$ J. Magnaudet, "High-Reynolds-number turbulence in a shear-free boundary layer: revisiting the Hunt-Graham theory," J. Fluid Mech. 484, 167-196 (2003).

${ }^{10}$ T. Uzkan and W. C. Reynolds, "A shear-free turbulent boundary layer," J. Fluid Mech. 28(4), 803-821 (1967).

${ }^{11} \mathrm{~S}$. Biringen and W. C. Reynolds, "Large-eddy simulation of the shear-free turbulent boundary layer," J. Fluid Mech. 103, 53-63 (1981).

${ }^{12}$ N. H. Thomas and P. E. Hancock, "Grid turbulence near a moving wall," J. Fluid Mech. 82(3), 481-496 (1977).

${ }^{13}$ D. T. Walker, R. I. Leighton, and L. O. Garza-Rios, "Shear-free turbulence near a flat free surface," J. Fluid Mech. 320, 19-51 (1996).

${ }^{14}$ E. L. G. Kit, E. J. Strang, and H. J. S. Fernando, "Measurement of turbulence near shear-free density interfaces," J. Fluid Mech. 334, 293-314 (1997).

${ }^{15}$ B. H. Brumley and G. H. Jirka, "Near-surface turbulence in a grid-stirred tank," J. Fluid Mech. 183, 235-263 (1987).

${ }^{16} \mathrm{M}$. W. McCorquodale, Interaction between oscillating-grid turbulence and a solid impermeable boundary, Ph.D. thesis, University of Nottingham (2017).

${ }^{17}$ B. J. Hopfinger and J. A. Toly, "Spatially decaying turbulence and its relation to mixing across density interfaces," J. Fluid Mech. 78(1), 155-175 (1976).

${ }^{18} \mathrm{~T}$. J. McDougall, "Measurements of turbulence in a zero-meanshear mixed layer," J. Fluid Mech. 94(3), 409-431 (1979).
${ }^{19}$ S. M. Thompson and J. S. Turner, "Mixing across an interface due to turbulence generated by an oscillating grid," J. Fluid Mech. 67(2), 349-368 (1975).

${ }^{20}$ W. H. M. Wan Mohtar and R. J. Munro, "Threshold criteria for incipient sediment motion on an inclined bedform in the presence of oscillating-grid turbulence," Phys. Fluids 25, 015103 (2013).

${ }^{21}$ N. S. Cheng and A. W. K. Law, "Measurements of turbulence generated by oscillating grid," J. Hydraul. Eng. 127, 201-208 (2001).

${ }^{22}$ S. Lucas, F. Moulin, and K. Guizien, "Oscillating grid mesocosm for studying oxygen dynamics under controlled unsteady turbulence," Limnol. Oceanogr.: Methods 14, 1-13 (2016).

${ }^{23} \mathrm{~A}$. Al-Homoud and M. Hondzo, "Energy dissipation estimates in oscillating grid setup: Ldv and piv measurements," Environ Fluid Mech 7, 143-158 (2007).

${ }^{24}$ H. J. S. Fernando and I. P. D. De Silva, "Note on secondary flows in oscillating grid, mixing box experiments," Phys. Fluids 5, 1849-1851 (1993).

${ }^{25}$ I. P. D. De Silva and H. J. S. Fernando, "Oscillating grids as a source of nearly isotropic turbulence," Phys. Fluids 6, 2455-2464 (1994).

${ }^{26}$ J. O. Hinze, Turbulence, 2nd ed. (McGraw-Hill, 1975).

${ }^{27}$ S. B. Pope, Turbulent Flows, 1st ed. (Cambridge University Press, 2000).

${ }^{28}$ J. C. R. Hunt and J. F. Morrison, "Eddy structure in turbulent boundary layers," Eur. J. Mech. B - Fluids 19, 679-694 (2000).

${ }^{29}$ J. D. A. Walker, C. R. Smith, A. W. Cerra, and T. L. Doligalski, "The impact of a vortex ring on a wall," J. Fluid Mech. 181, 99140 (1987).

${ }^{30} \mathrm{P}$. Orlandi and R. Verzicco, "Vortex rings impining on walls: axisymmetric and three-dimensional simulations," J. Fluid Mech. 256, 615-646 (1993).

${ }^{31}$ R. J. Munro, N. Bethke, and S. B. Dalziel, "Sediment resuspension and erosion by vortex rings," Phys. Fluids 21, 046601 (2009). 